\title{
Regenerative medicine: a new dawn for male reproductive issues
}

\begin{abstract}
The 'Regenerative medicine' holds the promise of engineering damaged tissues and organs by stimulating the body's own repair mechanisms to functionally heal previously irreparable tissues or organs. It includes the possibility of growing tissues and organs in the laboratory and implanting them when the body cannot heal itself. It has the potential to eliminate the issues of organ transplant rejection, and the issues of the shortage of organs available for donation by regenerating organs' cells from the patient's own tissue or cells. Contemplating on these peculiar qualities of Regenerative Medicine, it seems to have probably revolutionized the mode of treatment or therapy in almost every branch of medicines viz., cardiac, neurological, opthalmic, reproductive disorders etc. Therefore, based on reports from the relevant sources, the present article concisely compiles and retrospects the prospective implications/ applications of stem cells in treating male reproductive issues.
\end{abstract}

Keywords: azoospermia, male infertility, stem cell therapy, primordial germ cells, spermatogonial stem cells, sperms
Volume 3 Issue I - 2017

\author{
Pramod Vishwanath Prasad,' Abhay Kumar, ${ }^{2}$ \\ Zaved Ahsan Ansari, ${ }^{3}$ Kakali Purkayastha, ${ }^{4}$ \\ Meeta Kumari, ${ }^{5}$ Mayadhar Barik ${ }^{6}$ \\ 'Center for Biomedical Research, The Rockefeller University, \\ USA \\ ${ }^{2}$ Transplant Immunology \& Department of Microbiology, Indira \\ Gandhi Institute of Medical Sciences, Patna, Bihar, India \\ ${ }^{3}$ Department of Anatomy, All India Institute of Medical Sciences, \\ New Delhi-I I 0 029, India \\ ${ }^{4}$ Department of Paediatrics, All India Institute of Medical \\ Sciences, New Delhi, India \\ 'Patna Womens' College, Patna, Bihar, India \\ ${ }^{6}$ Department of Nuclear Medicine,All India Institute of Medical \\ Sciences, New Delhi, India
}

\begin{abstract}
Correspondence: Pramod Vishwanath Prasad, Center for Biomedical Research, The Population Council, The Rockefeller University, 1230, York Avenue, New York, NY 10065, USA, Email pkbiochem@yahoo.com,drpramod.pusa@gmail.com
\end{abstract}

Received: August 22, 2017 | Published: September 28, 2017
Abbreviations: ESC, embryonic stem cells; IPSC, induced pluripotent stem cells; SSC, spermatogonial stem cells; ART, assisted reproductive technology; IVF, in vitro fertilization; POF, porcine ovarian fibroblasts

\section{Introduction}

In 1992, the term 'regenerative medicine' was first used in an article on hospital administration by Leland Kaiser. He stated that, "A new branch of medicine will develop that attempts to change the course of chronic disease and in many instances will regenerate tired and failing organ systems". ${ }^{1,2}$ However, the widespread use of the term regenerative medicine is attributed to the founder of Human Genome Sciences, William A Haseltine. ${ }^{3}$ He recognized that these cells' have the potential to develop into a new kind of regenerative therapy as they are equipped with unprecedented ability to differentiate into all the cell types of the human body (i.e., pluripotency). ${ }^{4-6}$ Thus, the 'Regenerative medicine' is a branch of translational research in tissue engineering and molecular biology which deals with the "process of replacing, engineering or regenerating human cells, tissues or organs to restore or establish normal function". ${ }^{7,8}$ Some of the biomedical approaches within this field involve the use of stem cells. ${ }^{9}$

Stem cells are undifferentiated cells. They are capable of reproducing themselves i.e., self-renewal and differentiating into many different cell types, which can produce at least one type of highly differentiated descendant. ${ }^{10,11}$ They are highly specialised cells and different from all other types of cells. These cells are capable to replicate through cell division, even after longtime of dormancy. ${ }^{10,11}$ Scientists have found ways to manipulate stem cells into forming specific tissues or organs within the body. There are different types of stem cells. ${ }^{10}$ The majority of research focuses on Embryonic Stem Cells (ESC) because these cells can give rise to any other type of cell. This makes ESC malleable under certain conditions. Amniotic fluid, embryos, foetus, placenta, and umbilical cord blood contain ESCs. Stem cell research needs the use of human embryos so it often faces controversies. ${ }^{8,11,12}$ The stark difference between blastocyst-derived pluripotent stem cells and multipotent stem cells from adult organs is the number of types of differentiated cells that can be produced. Embryonic stem cells are derived from the inner cell mass of the blastocysts. The pluripotency and growth potential of ESC is uncomparable and beyond imagination. ${ }^{10-12}$ Embryonic stem cells of the mammalian blastocyst give rise to all the tissue lineages that initiate to emerge at gastrulation. These pluripotent cells can be propagated in vitro without loss of pluripotency. ${ }^{8,9,12,13}$ Due to these unique characteristics of ESC, research on ESC raises the possibility of 'designer' tissue and organ engineering. Many adult tissues harbor cells that do not complete their differentiation schedule. So, these cells act as selfrenewing stem cells whose normal fate is to regenerate site-specific tissue, in response to either physiological cell turnover or damage inflicted by injury or disease.$^{8,10,14-17}$ Bone marrow, muscle, and neural stem cells possess developmental potency much greater than their normal lineage-restricted fate. The developmental plasticity of adult stem cells, particularly those of bone marrow, make them potentially useful for replacing tissues, via transplantation or construction of bioartificial tissues, that either do not regenerate naturally or are damaged beyond their natural capability for regeneration. ${ }^{8,14-17}$ From the available reports, tissue regeneration and stem cell differentiation have been found to be influenced by the biomolecules like growth factors, micro RNAs, Extracellular Signal-Related Kinase (ERK); ${ }^{18}$ and biomechanical properties of few of the nanoparticles (dendrimers, liposomes, polymer-based nanoparticles, micelles, carbon nanotubes etc.). ${ }^{18-23}$ In addition to these two ways of replacing tissue, a third 
strategy of regenerative medicine is to stimulate regeneration in vivo from resident stem cells. ${ }^{8,10,14-17}$

Male reproductive health and fecundity is exclusively dependant on the normal development and maturation of specialized germ cells known as spermatozoa (sperm). In males, many cases of infertility have been categorized as unexplained. There are diseases that have been shown to cause loss of these germ cells, and deletions in critical genes on the $\mathrm{Y}$ chromosome. Additionally, infertility is also caused by an exposure to radiation, chemotherapy, and endocrine disruptors among other toxins. ${ }^{10,24}$

Globally, there is a lack of accurate statistics on rates of male infertility arising due to azoospermia, a condition in which sperms are not produced at all. However, Agarwal and his colleagues have estimated that infertility affects about $15 \%$ of couples globally (nearly 48.5 million couples). Among them, males are found to be solely responsible for $20-30 \%$ of infertility cases. ${ }^{25}$ However, Kumar and Singh reported that approximately $40-50 \%$ of all infertility cases are due to "male factor" infertility and as many as $2 \%$ of all men exhibit suboptimal sperm parameters. ${ }^{26}$ Although, this number does not accurately represent all regions of the world ${ }^{25}$ but infertility has insidious impact on the infertile individuals, their families, and society, which extend far beyond the inability to have a biological child. Lifestyle changes, fertility treatments, and assisted reproductive technology (ART) are available to help many infertile couples achieve their reproductive goals. However, all of these technologies require that the infertile individual is able to produce at least a small number of functional gametes viz., sperms in men. It is impossible for an azoospermic person to have a biological child. ${ }^{27}$ This review article emphasises on the infertile men and describes stem cell-based methods that are in the research pipeline and have an immense potential to provide a new fertility treatment options for men with azoospermia.

\section{Stem cells in male reproduction}

It is well established fact that a stem cell population is present in the male germ line, providing the basis for life-long production of male germ cells (i.e., precursor cells of spermatozoa or sperms) in the testes. The male germ line stem cells, or spermatogonial stem cells (SSC), are the only cell type in the body that can self-renew and transmit the genetic information to the next generation. Thus, these cells play pivotal role in preserving and maintaining the male fecundity. ${ }^{28-30}$ However, there are some iatrogenic, pathological and environmental factors which disrupt the normal course of SSC development leading to azoospermia, a major cause of male infertility. Therefore, in the reproductive medicine, reproducing germ cell development has been a key goal which can be accomplished by the combined efforts of both the reproductive biologists and stem cell technologists. ${ }^{31}$ Burgeoning research has shown that induced pluripotent stem cells (IPSC) can be derived from any tissue of the body using a cocktail of reprogramming factors. ${ }^{32,33}$ Several teams of scientists have reported that rodent, monkey, and human pluripotent ESC or IPSC can be differentiated into germ cells..$^{13,34-46}$ Recently, human ESC have been stimulated to form spermatozoon-like cells, which could potentially treat azoospermic males. ${ }^{47}$ In another study, culture of human ESC in mitotically inactivated porcine ovarian fibroblasts (POF) caused differentiation into germ cells, which got authenticated by the gene expression analysis. ${ }^{48}$ Moreover, investigators have carried out a robust, stepwise process that not only coaxed ESC from mice to turn into functional sperm-like cells, but also injected them into egg cells to produce fertile mouse offspring. These work have provided a hope in cases of male infertility. ${ }^{31}$

Like all other somatic cells, the 'primordial germ cells' are diploid and the common origins of spermatozoa in males and oocytes in females. So they represent the ancestors of the germline. In human embryos, they are already found in the primary ectoderm (epiblast) during the second week of development. ${ }^{49}$ In 2011, Hayashi and his colleagues successfully transformed mouse ESC into cells similar to primordial germ cells. Moreover, they have shown the feasibility of differentiating ESC or IPSC into epiblast-like cells which gave rise to primordial germ cell-like cells when cultured in the presence of Bone morphogenic protein-4 (BMP4).$^{13}$ When these resulting germ cells were transplanted into the seminiferous tubules of infertile male mice then they reinitiated spermatogenesis and formed haploid gametes. These gametes were used to fertilize mouse oocytes by Intra cytoplasmic sperm injection (ICSI). The embryos were transferred to recipient female mice and live offspring took birth. However, some unfavorable results forced to optimize the culture and differentiation protocols. ${ }^{13}$ In addition to this, they also reported generation of epiblast-like cells and primordial germ cell-like cells from femaleIPSC. The resulting primordial germ cell-like cells were transplanted into recipient females carrying functional eggs and live offsprings were obtained. ${ }^{50}$ In 2016, Cyranoski reported in Nature News about the preparation of artificial mouse sperm and successful replication of primordial germ cells. These cells were able to form non-motile immature sperm-like cells. In the IVF treatment, these sperm-like cells were injected into mice eggs, the fertile healthy mice were born. Throughout the experiment, researchers had the opportunity to witness meiosis in vitro ${ }^{51}$

Upon injection of cultured primordial germ cells into infertile mice testes, in fact into the seminiferous tubules of mice where the animals' sperm production takes place, the primordial germ cells further developed into mature spermatozoa and the female mice went on to produce healthy offspring. This research also formed the foundations for the study that the stem cells can be turned into spermatozoa. ${ }^{30}$ Moreover, a core research finding that ESC can develop into any cell type so it does not seem impossible that spermatozoa can also be developed from these cells. Recently published Chinese study utilized ESC from infertile male mice to create viable sperm capable of fertilisation. They were able to recreate working sperm cells. Although the sperm cells not being fully mature, they were active enough to father fertile offspring after successful IVF treatment in female mice. ${ }^{12}$ Now scientists are hopeful to replicate these results in humans. With these advances in human ESC research, infertile men may become fertile. Recently, two groups of researchers viz., Irie et al..$^{44}$ and Sasaki et al. ${ }^{46}$ reported the differentiation of human pluripotent stem cells into putative human primordial germ cell-like cells, exhibiting gene expression patterns similar to bonafide human primordial germ cells. Unfortunately, the human studies cannot be validated by transplantation or the production of offspring. Therefore, a surrogate assay of human spermatogenic potential is indispensable.

Couple of studies have elucidated that sperm stem cells require fertility genes. Investigators have determined that the reproductive homeobox (RHOX) family of transcription factors (i.e., regulatory proteins) that activate some genes and inactivate others, drive the development of stem cells into male germ cells in testes of mice. The investigators were also successful in linking RHOX gene mutations to male infertility in humans..$^{52,53}$ These studies also indicated that 
if fertility genes responsible for converting stem cells into sperms, activated properly, then resulting sperms can proove to be a boon for male infertile patients. This study clearly demonstrated the importance of genetics in spermatogenesis, but also suggested that some of these limitations could potentially be overcome by using stem cell technologies.

Recently published astonishing fact from the Stanford University School of Medicine and Montana State University showed that the adult infertile men's skin cells when used to create IPSC, they gave rise to primordial germ cells too. ${ }^{54}$ These cells normally turned into sperms, when transplanted into the reproductive system of male mice. These findings suggested that the infertile men may have had at least a few functioning germ cells as newborns or infants. Although more investigation needs to be done by collecting and freezing some of this tissue from young boys known to have $Y$ chromosome-linked mutation preventing the production of sperm. Such boys may have an option to have their own children later in life. Therefore, this research has also provided a ray of hope from the stem cell therapy in the treatment of azoospermia, the most severe form of male factor infertility. ${ }^{54}$

Feasibility of generating functional, sperm-producing germ cells from human ESC grown under defined conditions has been also described by Panula et al. ${ }^{40}$ However, those stem cells were made from human embryos that had been donated for research after IVF procedures. Therefore, they were not useful for couples wishing to have their own genetic children because the child would inherit a portion of the genome of the embryo from which the cells were derived. Furthermore, the process required scientists to engineer the stem cells so they would over-expression several genes, which are necessary to drive the ESC to become germ cells. On the contrary, the stem cells made from adult skin required no artificial manipulation. Once implanted into the testes, they differentiated into 'germ cell-like cells', simply by virtue of the environment in which they were placed. The cells expressed many genes known to be expressed in primordial germ cells, and underwent a genetic reprogramming process called demethylation associated with sperm production. ${ }^{34}$

Stem cells from the fertile and infertile men exhibited stark differences. It was estimated that the cells from the infertile men, who had a mutation in a region AZF1 genome, were about 50- to 100fold less efficient than fertile men in their ability to form primordial germ cells. Moreover, it provided very intriguing possibilities for men rendered sterile after cancer treatments. However, efficient conversion of skin cells into sperm arose hope for infertile men to become biological fathers. ${ }^{30,54}$

Infertility is one of the most common and devastating complications of cancer treatments, especially for young boys and men. ${ }^{30}$ The SSC are an important resource to preserve male fertility, particularly for childhood cancer patients who undergo potentially sterilizing cancer therapy before or around the time of puberty. Thus, it can be envisaged that SSC can be harvested before the therapy, cryopreserved, and following stem cell expansion and isolation, transplanted back to a patient after the therapy. Therefore, it can be anticipated that male germ line stem cells can be effectively utilized for male fertility preservation and restoration by surgical and pharmacological approaches. At present, the best and recommended approach to preserve fertility is to obtain and freeze gametes or tissue before the initiation of therapy that can damage or eliminate germ cells. ${ }^{28,29}$ However, if the IPSC to germ cell differentiation technology is responsibly developed and translated to the human clinic, this fertility preservation paradigm could change.
An adult survivor of a childhood cancer who desires to start his family and discovers that he is infertile could theoretically produce sperm and biological offspring from his own skin, blood, or other somatic cell type (Figure 1). This fertility preservation scenario can be applied not only to childhood cancer survivors, but all survivors or other infertile patients who cannot preserve or produce functional gametes. ${ }^{27}$

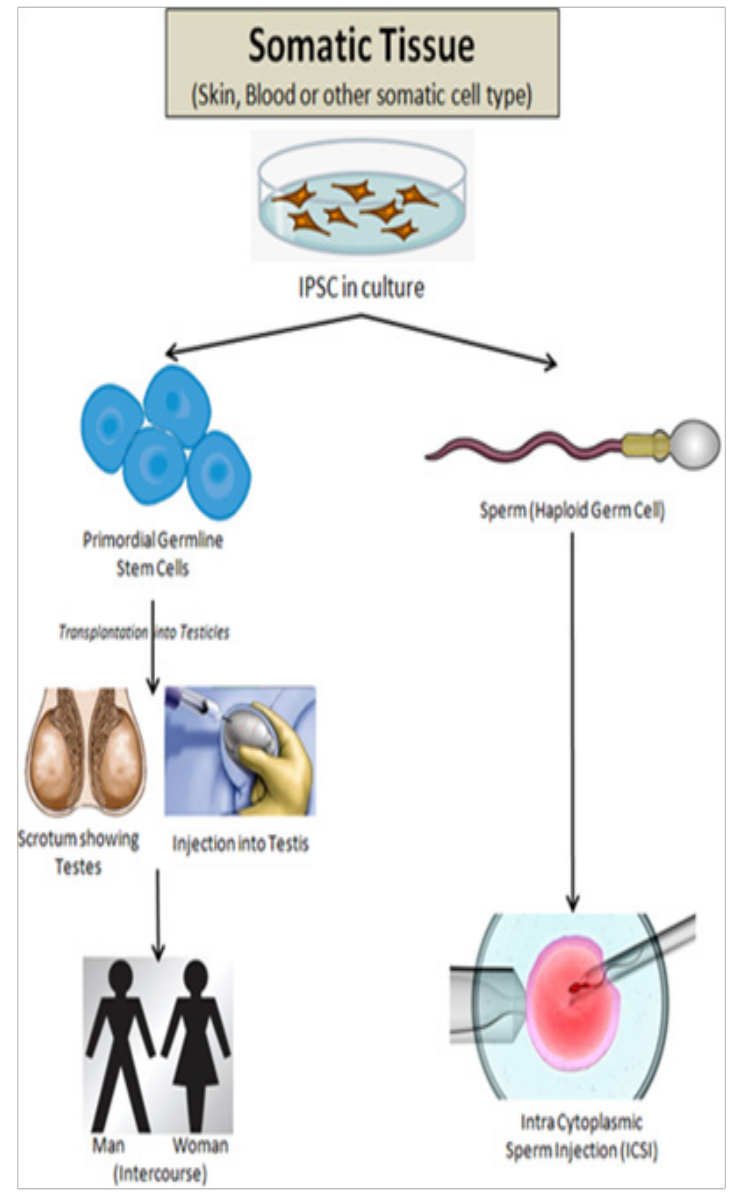

Figure I Patient specific IPSC can be derived from the patient somatic tissues and differentiated into germline stem cells, followed by transplantation into patient testes. This method seems to have potential to re-establish spermatogenesis and natural fecundity. It may also be feasible to differentiate IPSC directly into sperm that can be used to fertilize eggs by ICSI.

As the cancer chemotherapy drugs often work by destroying rapidly dividing cells they also kill other normally dividing cells in vicinity, including those that produce sperm. So some men choose to freeze sperm samples before therapy so they can use them for artificial insemination at a later date, but this is not an option for boys who have not yet attained puberty. Some investigators upon careful contemplation found that while boys don't make sperm cells, they do possess SSC that will eventually produce them. These SSC could be used to restore fertility, if cryopreserved during prepubertal stage. So Gassei et al. ${ }^{27}$ froze SSC samples from the testes of prepubescent and adult male Rhesus macaques. The monkeys were then given chemotherapy agents known to shut down sperm production. Few months later, the researchers injected each monkey's own SSC back into its testes. Subsequently, the sperm production was re-established in $75 \%$ of the adult animals and started normally in $60 \%$ of the prepubescent animals once they attained maturity. The resulting sperm 
were used to fertilise eggs and produced healthy embryos. Therefore, the scientists found the best option they have ever had. They have been able to successfully demonstrate this in a clinical setting with a species genetically very similar to human. Hence, few centres in the Europe and United States are already banking testicular tissue for boys in the hope that new stem cell-based therapies will become available. Moreover, a man could have his own stem cells transplanted, thus getting an opportunity to have children via natural intercourse. ${ }^{55}$

\section{Conclusion}

Till now, we have got only trivial understanding about the stem cells, even then the potential implications of stem cells in the Reproductive Biology and Medicine are apparent. There needs to resolve a number of basic research issues, before making these approaches a clinical reality. All aforementioned information provides a new insight into stem cell research that may have implications for human male infertility. Although the mice experiments have been groundbreaking but the scientific community is viewing the results with caution. Currently, human clinical applications seem to be remote. Germ cells of humans and mice develop differently. Nevertheless, the concept is still encouraging. Several new studies are being already planned and underway to further explore the applicability of stem cell based therapy to treat male infertility. This area of scientific endeavour holds great hope for infertile men wanting to father their own children. There seems to be every possibility that human male infertility can be reversed with stem cell research. Thus, new advancements in stem cell research may mean a breakthrough in the treatment of future male infertility,

\section{Acknowledgements}

We would like to greatly acknowledge Mr. Ramchandra Pokale, Chief Artist, CCM, AIIMS, New Delhi-29 who created the lucid illustration in Figure-1. Moreover, we would like to extend our heartfelt thanks to the anonymous reviewer(s) who helped us to improve the quality of our manuscript presentation. This work was not supported and funded by any financial grant from anywhere.

\section{Conflict of interest}

The authors declare that there is no conflict of interests regarding the publication of this manuscript.

\section{Authors contribution}

Pramod Vishwanath Prasad, Abhay Kumar, Kakali Purkayastha and Mayadhar Barik have contributed equally to this work.

\section{References}

1. Kaiser LR. The future of multihospital systems. Top Health Care Financ. 1992;18(4):32-45.

2. Lysaght MJ, Crager J. Origins. Tissue Eng Part A. 2009;15 (7):14491450 .

3. Viola J, Lal B, Grad O. The emergence of tissue engineering as a research field. National Science Foundation Arlington, VA, USA: Springer; 2003

4. Bailey Ronald. Liberation Biology: The Scientific and Moral Case for the Biotech Revolution. USA: Prometheus Books; 2005. 332 p.

5. Alexander Brian. Don't Die, Stay Pretty: The exploding science of superlongevity. Wired; 2000.
6. Haseltine WA. The emergence of regenerative medicine: a new field and a new society. Journal of Regenerative Medicine. 2004;2(4):17-23.

7. New health facility aims to translate stem cell science into therapies. USA: USC News; 2014

8. Mason C, Dunnill P. A brief definition of regenerative medicine. Regen Med. 2008;3(1):1-5.

9. Riazi AM, Kwon SY, Stanford WL. Stem cell sources for regenerative medicine. Methods Mol Biol. 2009;482:55-90.

10. Gargett CE. Stem cells in human reproduction. Reprod Sci. 2007;14(5):405-424

11. Du H, Taylor HS. Stem cells and female reproduction. Reprod Sci. 2009;16(2):126-139.

12. Zhou Q, Wang M, Yuan Y, et al. Complete meiosis from embryonic stem cell-derived germ cells in vitro. Cell Stem Cell. 2016;18(3):330-340.

13. Hayashi K, Ohta H, Kurimoto K, et al. Reconstitution of the mouse germ cell specification pathway in culture by pluripotent stem cells. Cell. 2011;146(4):519-532.

14. Health News - UM Leads in the Field of Regenerative Medicine: Moving from Treatments to Cures. University of miami, USA: Springer; 2014.

15. Regenerative Medicine. NIH Fact sheet, USA: Springer; 2006.

16. Regenerative medicine glossary. Regen Med. 2009;4(4 Suppl):S1-88.

17. Du H, Taylor HS. Stem cells and reproduction. Curr Opin Obstet Gynecol. 2010;22(3):235-241.

18. Dai R, Wang Z, Samanipour R, et al. Adipose-derived stem cells for tissue engineering and regenerative medicine applications. Stem Cells Int. 2016;2016:6737345.

19. Li B, Wang H, Qiu G, et al. Synergistic effects of vascular endothelial growth factor on bone morphogenetic proteins induced bone formation in vivo: influencing factors and future research directions. Biomed Res Int. 2016;2016:2869572.

20. van Rijt S, Habibovic P. Enhancing regenerative approaches with nanoparticles. J R Soc Interface. 2017;14(129).

21. Subbiah R, Hwang MP, Du P, et al. Tunable crosslinked cell-derived extracellular matrix guides cell fate. Macromol Biosci. 2016;16(11):17231734.

22. Zhang T, Lin S, Shao X, et al. Effect of matrix stiffness on osteoblast functionalization. Cell Prolif. 2017;50(3)

23. Tijore A, Cai P, Nai MH, et al. Role of cytoskeletal tension in the induction of cardiomyogenic differentiation in micropatterned human mesenchymal stem cell. Adv Healthc Mater. 2015;4(9):1399-1407.

24. Giwercman A, Rylander L, Lundberg GY. Influence of endocrine disruptors on human male fertility. Reprod Biomed Online. 2007;15(6):633642 .

25. Agarwal A, Mulgund A, Hamada A, et al. A unique view on male infertility around the globe. Reprod Biol Endocrinol. 2015;13:37.

26. Naina K, Singh AK. Trends of male factor infertility, an important cause of infertility: A review of literature. J Hum Reprod Sci. 2015;8(4):191196.

27. Gassei K, Orwig KE. Experimental methods to preserve male fertility and treat male factor infertility. Fertil Steril. 2016;105(2):256-266.

28. Pfeifer S, Goldberg J, Lobo R, et al. Fertility preservation in patients undergoing gonadotoxic therapy or gonadectomy: a committee opinion. Fertil Steril. 2013;100(5):1214-1223. 
29. Loren AW, Mangu PB, Beck LN, et al. Fertility preservation for patients with cancer: American society of clinical oncology clinical practice guideline update. J Clin Oncol. 2013;31(19):2500-2510.

30. Volarevic V, Bojic S, Nurkovic J, et al. Stem cells as new agents for the treatment of Infertility: current and future perspectives and challenges. Biomed Res Int. 2014;2014:507234.

31. Brazier Yvette. Sperm created from stem cells offer hope in cases of male infertility. Medical news today; 2016.

32. Takahashi K, Yamanaka S. Induction of pluripotent stem cells from mouse embryonic and adult fibroblast cultures by defined factors. Cell. 2006;126(4):663-676.

33. Takahashi K, Tanabe $\mathrm{K}$, Ohnuki $\mathrm{M}$, et al. Induction of pluripotent stem cells from adult human fibroblasts by defined factors. Cell. 2007;131(5):861-872

34. Kee K, Angeles VT, Flores M, et al. Human DAZL, DAZ and BOULE genes modulate primordial germ-cell and haploid gamete formation. $\mathrm{Na}$ ture. 2009;462(7270):222-225.

35. Easley CA, Phillips BT, McGuire MM, et al. Direct differentiation of human pluripotent stem cells into haploid spermatogenic cells. Cell Rep. 2012;2(3):440-446.

36. Kee K, Gonsalves JM, Clark AT, et al. Bone morphogenetic proteins induce germ cell differentiation from human embryonic stem cells. Stem Cells Dev. 2006;15(6):831-837.

37. Teramura $\mathrm{T}$, Takehara $\mathrm{T}$, Kawata $\mathrm{N}$, et al. Primate embryonic stem cells proceed to early gametogenesis in vitro. Cloning Stem Cells. 2007;9(2):144-156.

38. Park TS, Galic Z, Conway AE, et al. Derivation of primordial germ cells from human embryonic and induced pluripotent stem cells is significantly improved by coculture with human fetal gonadal cells. Stem Cells. 2009;27(4):783-795.

39. Yamauchi K, Hasegawa K, Chuma S, et al. In vitro germ cell differentiation from cynomolgus monkey embryonic stem cells. PloS One. 2009;4(4):e5338.

40. Panula S, Medrano JV, Kee K, et al. Human germ cell differentiation from fetal- and adult-derived induced pluripotent stem cells. Hum Mol Genet. 2011;20(4):752-762.

41. Dominguez AA, Chiang HR, SukhwaniM, et al. Human germ cell formation in xenotransplants of induced pluripotent stem cells carrying $\mathrm{x}$ chromosome aneuploidies. Sci Rep. 2014;4:6432.
42. Durruthy Durruthy J, Ramathal C, Sukhwani M, et al. Fate of induced pluripotent stem cells following transplantation to murine seminiferous tubules. Hum Mol Genet. 2014;23(12):3071-3084.

43. Ramathal C, Durruthy Durruthy J, Sukhwani M, et al. Fate of iPSCs derived from azoospermic and fertile men following xenotransplantation to murine seminiferous tubules. Cell Rep. 2014;7(4):1284-1297.

44. Irie N, Weinberger L, Tang WW, et al. Sox17 is a critical specifier of human primordial germ cell fate. Cell. 2015;160(1-2):253-268.

45. Ramathal C, Angulo B, Sukhwani M, et al. Ddx3y gene rescue of a y chromosome azfa deletion restores germ cell formation and transcriptional programs. Sci Rep. 2015;5:15041

46. Sasaki K, Yokobayashi S, Nakamura T, et al. Robust in vitro induction of human germ cell fate from pluripotent stem cells. Cell Stem Cell. 2015;17(2):178-194.

47. Ledford H. Sperm-like cells made from human embryonic stem cells. Nature News; 2009.

48. Richards M, Fong CY, Bongso A. Comparative evaluation of different in vitro systems that stimulate germ cell differentiation in human embryonic stem cells. Fertil Steril. 2008;93(3):986-994.

49. Nikolic A, Volarevic V, Armstrong L, et al. Primordial germ cells: Current knowledge and perspectives (Review Article). Stem Cells Int. 2016;2016:1741072.

50. Hayashi K, Ogushi S, Kurimoto K, et al. Offspring from oocytes derived from in vitro primordial germ cell-like cells in mice. Science. 2012;338(6109):971-975.

51. Cyranoski David. Researchers claim to have made artificial mouse sperm in a dish. Nature News; 2016.

52. Song HW, Bettegowda A, Lake BB, et al. The homeobox transcription factor RHOX10 Drives mouse spermatogonial stem cell establishment. Cell Rep. 2016;17(1):149-164.

53. Borgmann J, Tüttelmann F, Dworniczak B, et al. The human RHOX gene cluster: target genes and functional analysis of gene variants in infertile men. Hum Mol Genet. 2016;25(22):4898-4910.

54. Eisenberg Michael. Recent study found that Stem cells from some infertile men form germ cells when transplanted into mice; 2014.

55. Will Ferguson. Sperm stem cells restore male fertility. New Scientist Magazine, Journal reference: Cell Stem Cell. 2012. 